\title{
25-gauge vitrectomy and gas for the management of rhegmatogenous retinal detachment
}

\author{
Miroslav Veith ${ }^{\mathrm{a}}$, Zbynek Stranak ${ }^{\mathrm{a}}$, Martin Pencak ${ }^{\mathrm{a}}$, Jana Vranova ${ }^{\mathrm{b}}$, Pavel Studeny ${ }^{\mathrm{a}}$
}

\begin{abstract}
Aims. To evaluate the anatomical and functional results in patients with rhegmatogenous retinal detachment (RRD) who underwent 25-gauge pars plana vitrectomy (PPV) with gas tamponade.

Materials and Methods. A retrospective evaluation of 126 eyes of 126 patients (79 men, 47 women) with RRD who underwent 25-gauge PPV with gas tamponade (13\% C3F8 in 87 eyes, 20\% SF6 in 39 eyes). 113 patients (89.7\%), were operated on under local anaesthesia, 13 patients (10.3\%) under general anaesthesia. Macula was detached in 85 eyes (67.5\%). 53 eyes had pseudophakic RRD, 73 eyes were phakic. Anatomical success of the primary intervention, change in best corrected visual acuity (BCVA) and incidence of complications were assessed. An average follow-up period is 7.2 months (6-15).

Results. With single operation, retinal attachment was achieved in 125 eyes (99.2\%); the final anatomical success was $100 \%$. The initial mean BCVA was 0.89 logMar (2.00 to 0.00 ); at the end of the follow-up period, it improved to $0.23 \log M A R(1.00$ to -0.10$), P<0,0001$. During the first post-intervention day, hypotony of the eye below $10 \mathrm{mmHg}$ was observed in 1 patient $(0.8 \%)$; on the contrary, intraocular pressure was temporarily increased to $25 \mathrm{mmHg}$ and more in 36 patients (28.6\%).

Conclusion. The surgical treatment of RRD using 25-gauge PPV with expansive gas tamponade renders excellent anatomical results and improvement in BCVA. The incidence of complications and necessity of sclerotomy suturing are low.

Key words: 25-gauge, vitrectomy, retinal detachment, rhegmatogenous, gas, oblique, transconjunctival

Received: February 15, 2018; Accepted: June 7, 2018; Available online: June 21, 2018

https://doi.org/10.5507/bp.2018.034

${ }^{a}$ Department of Ophthalmology, University Hospital Kralovske Vinohrady and Third Faculty of Medicine, Charles University in Prague, Czech Republic

${ }^{b}$ Department of Medical Biophysics and Medical Informatics, Third Faculty of Medicine, Charles University in Prague, Czech Republic Corresponding author: Miroslav Veith, e-mail:mveith@email.cz
\end{abstract}

\section{INTRODUCTION}

Rhegmatogenous retinal detachment (RRD) is a serious, vision-threatening condition which affects approximately 1 out of 10.000 people every year. The development of RRD is caused by formation of a retinal break followed by accumulation of intraocular fluid in the subretinal space. Contrary to some other vitreoretinal diseases, RRD requires a prompt surgical intervention ${ }^{1,2}$. Current intervention methods used are scleral buckling, pneumatic retinopexy and pars plana vitrectomy (PPV), the latter being performed either as a sole procedure or combined with scleral buckling ${ }^{3-5}$.

The best surgical method is still being discussed. Treatment in often individualized based on surgeon experience and preferences, number and location of retinal breaks, amount of subretinal fluid, state of the macula, presence of proliferative vitreoretinopathy (PVR), condition of the lens, state of the vitreous body and overall condition of the patient.

The development of sutureless vitrectomy methods was a significant milestone in the vitreoretinal surgery. Such techniques offer benefits of a mini-invasive procedure with a higher post-operative comfort and faster recovery.
In this paper, anatomical and functional results of 25-gauge PPV for RRD are evaluated.

\section{MATERIALS AND METHODS}

We retrospectively evaluated medical records of 126 consecutive eyes of 126 patients with RRD. Patients underwent primary surgery for RRD in the Department of Ophthalmology of the Kralovske Vinohrady University Hospital from May 2013 through June 2016. 25-gauge PPV was performed using the Constellation vitrectomy machine (Alcon, Forth Worth, TX, USA), with the Ultravit vitrectomy probe with a cut rate of 5,000 cuts/ min. Resight 500 (Zeiss, Germany) was used to visualise the fundus. Patient with minimum follow-up 6 month were enrolled. None of the patients has PVR greater than grade $\mathrm{B}$.

All patients were operated by the same surgeon (MV). 113 patients $(89.7 \%)$ were operated under retrobulbar anaesthesia (Marcain), 13 patients (10.3\%) were operated under general anaesthesia. Cannulas were placed in a standard manner - inferotemporally for infusion cannula, superotemporally and superonasally (in phakic eyes, 4 $\mathrm{mm}$ posterior to the limbus, in pseudophakic eyes, 3.5 
$\mathrm{mm}$ posterior to the limbus). After displacing the conjunctiva, the trocar/cannula system was introduced parallel to the limbus under an angle of approximately $10^{\circ}$, with a simultaneous denting of the sclera. As soon as the cannula top touched the sclera, the introduction angle was changed to approximately $60^{\circ}$.

The procedure itself started with core vitrectomy. Meticulous peripheral vitreous removal was performed especially around retinal breaks in order to relieve vitreoretinal tractions, and also along the detached retina and around the outlets of cannulas in order to prevent the incarceration of the vitreous into the sclerotomies after the extraction of cannulas at the end of the surgery. The peripheral vitreous was removed in retroillumination using scleral indentation with light probe. In some cases, perfluorodecalin (Arcaline, Arcadophta, France) was applied to immobilise the detached retina and to facilitate the evacuation of subretinal fluid through peripheral breaks. The decision on its application was based on the detachment size, subretinal fluid quantity, and localisation and size of breaks. Perfluorodecalin was also applied in patients with detached macula when membrane peeling was indicated. If epiretinal membrane (ERM) was apparent in macula or where its presence was suspected, brilliant blue (Ocublue, Aurolab, India) dye was used and ERM and internal limiting membrane (ILM) peeling was performed. This procedure was followed by fluid-air exchange. Charles Flute Cannula (Alcon, Forth Worth, TX, USA) or vitrectomy probe was used to drain fluid from the subretinal space through the break, and subsequently, complete fluid-air exchange was performed. Complete retinal attachment was not required, where small volume of residual subretinal fluid was present after the complete fluid-air exchange, it was left. Retinopexy of margins of the break and lattice degenerations was performed under air using endolaser or cryotherapy probe. When perfluorodecalin was used, retinopexy was usually performed before the fluid-air exchange. If multiple retinal breaks and lattice degeneration were present, $360^{\circ}$ retinopexy was performed. Non-expansive concentration of sulfur hexafluoride (SF6) (Alchimia, Italy) or perfluoropropane (C3F8) (Alchimia, Italy) was used as a tamponade in all the patients. In patients with superior retinal breaks, 20\% SF6 was usually used; in patients with inferior retinal breaks, 13\% C3F8 was usually used. The choice of gas type also depended on its availability at the workplace. After the extraction of the cannulas, tightness of the sclerotomy was checked. If leakage was present, digital massage was performed. If digital massage was not sufficient to stop the leakage, sclerotomy was sutured using Vicryl 8-0 (Ethicon, Johnson \& Johnson Int). Patients were advised on a suitable head positioning during a one-week period depending on the location of breaks.

Patients were examined in our ophthalmology department one day after the intervention, one month, 2 months after the intervention, and then as needed. Only patients with follow up period six month and more were enrolled. In all the examinations, the best corrected visual acuity (BCVA) was checked using ETDRS charts (Early Treatment Diabetic Retinopathy Study), and it was converted to $\log$ MAR values for statistical purposes. Intraocular pressure (IOP) was measured, and an examination was performed using a slit lamp including a biomicroscopy of the fundus in artificial mydriasis.

Statistical analyses were performed using statistical software STATISTICA (version 12). For the purposes of statistical testing, the BCVA results were converted to $\log$ MAR equivalents. All the decisions were taken at a significance level (alpha) of 0.05 .

The anatomical success of the primary intervention was evaluated, as well as the final anatomical success, change in visual acuity, numbers of sutured sclerotomies and incidence of complications. An average follow-up period was 7.2 months (6-15).

\section{RESULTS}

126 eyes of 126 patients ( 79 men, 47 women) were included in the study. Average age was 61.1 years (23-81). Retina was detached in one quadrant in 12 eyes, in 2 to 3 quadrants in 100 eyes, and retina was completely detached in 4 eyes. Macula was detached in 85 eyes (67.5\%). One break was detected in 53 eyes $(42.1 \%), 3$ and more in 41 eyes $(32.5 \%)$. At least one break was present in inferior retinal quadrants between numbers 4-8 in 48 eyes (38.1\%). 53 eyes were pseudophakic and 73 phakic. During the

Table 1. Patient's demographics.

\begin{tabular}{lc}
\hline Total no. of eyes (total no. of patients) & $126 / 126$ \\
\hline Age, $(\mathrm{y})$ & \\
Mean \pm SD & $61.1 \pm 10.6$ \\
Median (range) & $62(23-81)$ \\
\hline Sex, no. (\%) & 79 \\
Male & 47 \\
Female & $7.2 \pm 5.4$ \\
\hline Symptoms duration (d) & $7(1-28)$ \\
Mean \pm SD & \\
Median (range) & $7.2 \pm 2.1$ \\
\hline Follow-up (mo) & $6(6-15)$ \\
Mean \pm SD & 12 \\
Median (range) & 62 \\
\hline Quadrant of RD, no. of eyes & 38 \\
1 & 14 \\
2 & \\
3 & $41(32.5 \%)$ \\
4 & $85(67.5 \%)$ \\
\hline Macula detachment, no. (\%) of eyes & \\
Macula on & $53(57.9 \%)$ \\
Macula off & $32.1 \%)$ \\
\hline Number of tears, no. of eyes & 41 \\
1 & \\
2 & \\
23 & \\
\hline Pens status (\%) & \\
Pseudophakic & \\
\hline & \\
\hline
\end{tabular}

$\mathrm{RD}$, retinal detachment 
Table 2. Visual acuity results.

\begin{tabular}{lccc}
\hline & $\begin{array}{c}\text { Preoperative VA, } \\
\text { logMAR } \\
\text { (Snellen VA) }\end{array}$ & $\begin{array}{c}\text { Postperative VA, } \\
\text { logMAR }\end{array}$ & $P$ \\
\hline Snellen VA) & \\
Macula-on RD & $0.36(0.62)$ & $0.19(0.81)$ & 0.002 \\
All RD & $0.89(0.35)$ & $0.23(0.71)$ & $<0.0001$ \\
\hline
\end{tabular}

VA, Visual Acuity; RD, Retinal Detachment

follow-up period, cataract surgery was performed in 31 of 73 phakic patients $(42.5 \%)$ (Table 1$)$.

Perfluorodecalin was used in 62 eyes $(49.2 \%)$, and in 19 eyes (15.1\%) ERM and ILM peeling was performed. $360^{\circ}$ retinopexy was performed in 14 patients $(11.1 \%)$. $13 \% \mathrm{C} 3 \mathrm{~F} 8$ was used as a tamponade in 87 eyes (69\%), 20 $\%$ SF6 in 39 eyes (31\%).

After the primary surgery, retinal attachment was achieved in 125 eyes (99.2\%). In one eye, another PPV with gas tamponade was performed within one month due to a persistent flat retinal detachment in the periphery of the inferior temporal quadrant. The final anatomical success rate of the whole group was $100 \%$. Mean initial BCVA was 0.89 logMar (2.00 to 0.00); at the end of the follow-up period, it improved to $0.23 \log$ MAR (1.00 to -0.10), $P<0.0001$ (Table 2).

In one case, at the end of the surgery, one sclerotomy had to be sutured due to leakage; in one case, two sclerotomies had to be sutured, and in a case of one patient, all three sclerotomies had to be sutured. On the first postoperation day, a mean IOP value was $21 \mathrm{mmHg}(9-45)$. One eye was hypotonic - less than $10 \mathrm{mmHg}(0.8 \%)$; intraocular pressure was $9 \mathrm{mmHg}$. In 36 patients $(28.6 \%)$ intraocular pressure was temporarily increased to $25 \mathrm{mmHg}$ and more $(25-30 \mathrm{mmHg}$ in 23 eyes, $31-40 \mathrm{mmHg}$ in 12 eyes, over $41 \mathrm{mmHg}$ in 1 eye). The intraocular pressure normalized with topical anti-glaucoma therapy. During the follow-up period, one patient developed cystoid macular oedema (the eye had suffered an injury and it had been intervened twice for strabismus), and one patient developed ERM. In one patient with high myopia and loosened suspensory ligaments of the lens, gas penetrated into the anterior chamber, causing a pupillary block and an elevation of the IOP. When laser iridotomy was performed, IOP values returned to normal. No other complications including endophthalmitis were observed.

\section{DISCUSSION}

The first generation of 25-gauge PPV was presented by Fuji in 2002 (ref. ${ }^{6}$ ). Instruments used, however, were flexible and not suitable for surgical solution of pathologies which required a more complex peripheral vitrectomy and major eye handling. It was also associated with higher risk of peri- and postoperative complications ${ }^{7}$. In 2010, the second generation instrumentation was introduced, offering better fluid dynamics, improved rigidity of tools and a wide-field illumination. It quickly became a preferred technique for surgeons who began to use it not just for the solution of vitreous opacities and macular diseases, but also in cases with more complex vitreoretinal pathologies $^{8-14}$.

25-gauge PPV offers many advantages, such as a mini-invasive approach, shortened intervention time, lower post-operative inflammatory reaction and patient's discomfort, minor conjunctival scarring and minor corneal astigmatism ${ }^{9,15}$. Cryosurgical approach is still useful in RRD management, especially in phakic patients ${ }^{3,5}$. However, PPV is more suitable for patients suffering from multiple breaks in different quadrants, bullous retinal detachment, breaks extending post-equatorially, breaks with noticeable vitreoretinal traction, in patients with an unclear situation as to break borders (no breaks detected preoperatively or impossibility to identify with certainty all the breaks during the pre-operative assessment) and in pseudophakic patients ${ }^{3}$. With PPV, vitreoretinal tractions may be relieved, and all the retinal breaks may be precisely identified and repaired. The PPV may also help to avoid some serious complications related to scleral buckling ${ }^{3,15,16}$. Cryosurgical interventions also tend to be longer and general anaesthesia is required.

In our group, retinal attachment after primary operation was achieved in $99.2 \%$ of eyes. This result is comparable to those achieved by other techniques ${ }^{3-5}$. Similar results have been published by several authors ${ }^{7,11,14,15,17}$. On the contrary, Lai et al. achieved retinal attachment after the primary operation only in $74 \%$ of the operated eyes ${ }^{12}$. Operations were unsuccessful mainly in eyes with multiple retinal breaks; the difference, however, was not statistically significant probably due to the size of the studied group. Our group included 41 eyes (32.5\%) with three and more breaks, and in all the cases, retinal attachment was achieved after the primary surgery. In a case of one eye which required another intervention, there were two breaks in superior quadrants. As a standard, the abovementioned authors performed a $360^{\circ}$ thorough removal of the vitreous base. However, we perform just an extended core vitrectomy, and peripheral vitreous is meticulously removed only at the borders of retinal breaks and along the detached retina. We do not perform extensive peripheral vitrectomy at sites without obvious vitreoretinal tractions. This approach may reduce the risk of minor iatrogenic retinal breaks development which may easily remain undetected and become the reason for operation failure. In case of multiple retinal breaks and peripheral retinal degenerations, $360^{\circ}$ retinopexy was performed (in a total of 14 eyes), which could also contribute to a better anatomical success in our group. Other authors confirm this assumption. Acar et al. presented a group of 22 operated eyes, in which a meticulous $360^{\circ}$ removal of vitreous base was performed, and $360^{\circ}$ laser peripheral retinopexy was performed in all the eyes. In 21 eyes, primary retinal attachment was achieved (95.5\%) (ref. ${ }^{15}$ ). In the group of Miller et al. retinal attachment was achieved in all the eyes when $360^{\circ}$ laser retinopexy was performed; in comparison, when focal laser was applied, retinal attachment was achieved only in $76.9 \%$ of the eyes ${ }^{11}$. 
Operation success was achieved also in patients with retinal breaks localised in the inferior retinal quadrants. In 48 eyes (38.1\%), at least one break was detected between numbers 4 and 8 . Retinal attachment was achieved in all these patients after the primary surgery. Similar results were achieved by Duvdevan et al. ${ }^{16}$. In their series, $96 \%$ (24 eyes out of 25 ) primary retinal attachment was achieved in patients with retinal breaks in inferior quadrants; compared to $82.4 \%$ (28 out of 34 ) primary retinal attachment in the eyes with breaks in superior quadrants. Also, Dell 'Omo et al. were very successful in retinal detachment repair in eyes with retinal breaks in inferior quadrants; retinal attachment was achieved in $92.7 \%$ of the eyes using PPV with gas tamponade without scleral buckling ${ }^{18}$.

Proliferative vitreoretinopathy is a frequent cause of the RRD operation failure ${ }^{12,19}$. Mini-invasive 25-gauge vitrectomy causes a mild inflammatory response and a lower production of pro-inflammatory factors causing PVR. Despite this, $21 \%$ of patients in the group of Lai et al. developed post-operative PVR ( ref. $^{12}$ ). In our group, no patient developed PVR during the postoperative period. Also other authors reported low PVR incidence ${ }^{13,16,18,19}$. Such results may be explained with better fluid dynamics in the second-generation 25-gauge PPV, when pro-proliferative substances are more meticulously eliminated (e.g. retinal pigment epithelium cells, growth factors and cytokines).

Sclerotomy leakage with subsequent hypotony became rather frequent complication of sutureless PPV approaches. In our group, it was necessary to suture at least one sclerotomy in three eyes. On the first postoperative day, a hypotony under $10 \mathrm{mmHg}$ was detected only in 1 eye $(9 \mathrm{mmHg})$. The value of IOP returned to normal within two days without any necessity of further intervention and without complications. A low incidence of sclerotomy leakage and postoperative hypotony might be attributed to extra-oblique scleral incisions. A longer intrascleral tunnel provides better sclerotomy tightness. Hsu et al. observed a lower incidence of postoperative hypotony $\leq 5 \mathrm{mmHg}$ after 25-gauge PPV without tamponade in eyes with oblique scleral incisions compared to straight sclerotomies $(1.8 \%$ vs. $10 \%)\left(\right.$ ref. $\left.^{20}\right)$. Similarly, Acar et al. observed an incidence of hypotony under $8 \mathrm{mmHg}$ in $17.1 \%$ of the eyes when straight sclerotomies were performed and intraocular tamponade was not used ${ }^{21}$. Bourgault, however, did not prove that oblique sclerotomies decreased the incidence of postoperative hypotony compared to straight sclerotomies both in eyes with gas tamponade or without tamponade $(9.9 \%$ vs. $9.2 \% ; P=0.85)\left(\right.$ ref. $\left.^{22}\right)$. The application of gas tamponade, however, markedly decreased the risk of hypotony ( $4.8 \%$ vs. $20.0 \%$ in eyes without tamponade; $P=0.0001)$. Gas was also used for temporary tamponade in all the eyes in our group. Gas tamponade generates pressure on sclerotomies, which contributes to their better tightness and speeds up their healing. In our group of patients with idiopathic macular holes operated with 25 -gauge PPV and gas tamponade $(n=53)$, hypotony was not observed in any eye ${ }^{9}$. Furthermore, the vitrectomy without a thorough basectomy leads to a shorter operation time with minor sclerotomy contusion. This fact may also contribute to a better tightness of sclerotomies.

\section{CONCLUSION}

25-gauge PPV with gas tamponade is an efficient operation technique for the management of non-complicated retinal detachment including retinal breaks in inferior quadrants with a minimal incidence of complications. Extra-oblique sclerotomy incisions minimise the risk of postoperative hypotony and the necessity to suture sclerotomies.

\section{ABBREVIATIONS}

PPV, Pars plana vitrectomy; RRD, Rhegmatogenous retinal detachment; BCVA, Best corrected visual acuity; PVR, Prolipherative vitreoretinopathy; IOP, Intraocular pressure; ILM, Internal limiting membrane; ERM, Epiretinal membrane.

Author contributions: All co-authors contributed equally to preparing the manuscript. All co-authors also have read the final manuscript and accept its conclusions.

Conflict of interest statement: The authors state that there are no conflicts of interest regarding the publication of this article.

\section{REFERENCES}

1. Hejsek L, Dusova J, Stepanov A, Rozsival P. Scleral buckling for Rhegmatogenous retinal detachment. Cesk Slov Oftalmol 2014;70(3):110.

2. Chrapek O, Sin M, Jirkova B, Jarkovksy J, Rehak J. Functional results of cryosurgical procedures in rhegmatogenous retinal detachment including macula region - our experience. Cesk Slov Oftalmol 2013;69(5):202-6.

3. Chrapek O, Sin M, Jirkova B, Jarkovksy J, Rehak J. Anatomical results of cryosurgical procedures in rhegmatogenous retinal detachment - our experience. Cesk Slov Oftalmol 2013;69(4):164-8.

4. Lewis SA, Miller DM, Riemann CD, Foster RE, Petersen MR. Comparison of 20-, 23-, and 25-gauge pars plana vitrectomy in pseudophakic rhegmatogenous retinal detachment repair. Ophthalmic Surg Lasers Imaging 2011;42(2):107-13.

5. Hejsek L, Dusova J, Stepanov A, Rozsival P. Scleral buckling for rhegmatogenous retinal detachment. Cesk Slov Oftalmol 2014;70(3):1103.

6. Fujii GY, De Juan E Jr, Humayun MS, Pieramici DJ, Chang TS, Awh C, Ng E, Barnes A, Wu SL, Sommerville DN. A new 25-gauge instrument system for transconjunctival sutureless vitrectomy surgery. Opthalmology 2002;109(10):1807-12.

7. Mura M, Tan SH, De Smet MD. Use of 25 -gauge vitrectomy in the management of primary rhegmatogenous retinal detachment. Retina 2009;29(9):1299-304.

8. Hejsek L, Stepanov A, Dusova J, Marak J, Nekolova J, Jiraskova N, Codenotti M. Microincision 25G pars plana vitrectomy with peeling of the internal limiting membrane and air tamponade in idiopathic macular hole. Eur J Ophthalmol 2017;27(1):93-7.

9. Veith M, Stranak Z, Pencak M, Studeny P. Surgical Treatment of the Idiopathic Macular Hole by Means of 25-Gauge Pars Plana Vitrectomy with the Peeling of the Internal Limiting Membrane 
Assisted by Brilliant Blue and Gas Tamponade. Cesk Slov Oftalmo 2015;71(3):170-4.

10. Hejsek L, Kadlecova J, Dusova J, Machackova M, Jiraskova N. Pars Plicata Vitrectomy in Premature Newborns for Retinal Detachment as a Result of Retinopathy of Prematurity, our Results. Cesk Slov Oftalmol 2017;73(4):140-5.

11. Miller DM, Riemann CD, Foster RE, Petersen MR. Primary repair of retinal detachment with 25-gauge pars plana vitrectomy. Retina 2008;28(7):931-6.

12. Lai MM, Ruby AJ, Sarrafizadeh R, Urban KE, Hassan TS, Drenser KA, Garretson BR. Repair of primary rhegmatogenous retinal detachment using 25-gauge transconjunctival sutureless vitrectomy. Retina 2008;28(5):729-34.

13. Gotzaridis S, Liazos E, Petrou P, Georgalas I. 25-Gauge Vitrectomy and Incomplete Drainage of Subretinal Fluid for the Treatment of Primary Rhegmatogenous Retinal Detachment. Ophthalmic Surg Lasers Imaging Retina 2016:47(4):333-5.

14. Kunikata $\mathrm{H}$, Nishida K. Visual outcome and complications of 25-gauge vitrectomy for rhegmatogenous retinal detachment; 84 consecutive cases. Eye (Lond) 2010;24(6):1071-7.

15. Acar N, Kapran Z, Altan T, Unver YB, Yurtsever S, Kucuksumer Y. Primary 25-gauge sutureless vitrectomy with oblique sclerotomies in pseudophakic retinal detachment. Retina 2008:28(8):1068-74.

16. Duvdevan N, Mimouni M, Feigin E, Barak Y. 25-gauge pars plana vit- rectomy and SF6 for the repair of primary inferior rhegmatogenous retinal detachment. Retina 2016;36(6):1064-9.

17. Von Fricken MA, Kunjukunju N, Weber C, Ko G. 25-gauge sutureless vitrectomy versus 20-gauge vitrectomy for the repair of primary rhegmatogenous retinal detachment. Retina 2009;29(4):444-50.

18. Dell'Omo R, Barca F, Tan HS, Bijl HM, Oberstein SY, Mura M. Pars plana vitrectomy for the repair of primary, inferior rhegmatogenous retinal detachment associated to inferior breaks. A comparison of a 25-gauge versus a 20-gauge system. Graefes Arch Clin Exp Ophthalmol 2013;251(2):485-90.

19. Chen X, Zhang Y, Yan Y, Hong L, Zhu L, Deng J, Din Q, Huang Z, Zhou H. Complete subretinal fluid drainage is not necessary during vitrectomy surgery for macula-off rhegmatogenous retinal detachment with peripheral breaks: A Prospective, Nonrandomized Comparative Interventional Study. Retina 2017;37(3):487-93.

20. Hsu J, Chen E, Gupta O, Fineman MS, Garg SJ, Regillo CD. Hypotony after 25-gauge vitrectomy using oblique versus direct cannula insertions in fluid-filled eyes. Retina 2008;28(7):937-40.

21. Acar N, Kapran Z, Unver YB, Altan T, Ozdogan S. Early postoperative hypotony after 25-gauge sutureless vitrectomy with straight incisions. Retina 2008:28(4):545-52.

22. Bourgault $\mathrm{S}$, Tourville E. Incidence of postoperative hypotony in 25 -gauge vitrectomy: oblique versus straight sclerotomies. Can J Ophthalmol 2012;47(1):21-3. 